OPEN ACCESS

Edited by:

Nicola Silvestris,

University of Bari Aldo Moro, Italy

Reviewed by:

Azin Nahvijou,

Tehran University of Medical Science,

Sudabeh Alatab,

Tehran University of Medical Sciences,

Iran

${ }^{*}$ Correspondence:

Pedro Caruso

pedro.caruso@hc.fm.usp.br

Specialty section:

This article was submitted to

Cancer Epidemiology and Prevention,

a section of the journal

Frontiers in Oncology

Received: 23 July 2021 Accepted: 08 November 2021

Published: 30 November 2021

Citation:

Caruso P, Testa RS, Freitas ICL, Praça APA, Okamoto VN, Santana PV, Costa RT, Kawasaki AM, Fumis RRL,

Pino Illanes WA, Costa ELV,

Midega TD, Correa TD, de Carvalho FRT and Ferreira JC (2021)

Cancer-Related Characteristics Associated With Invasive Mechanical Ventilation or In-Hospital Mortality in Patients With COVID-19 Admitted to

ICU: A Cohort Multicenter Study.

Front. Oncol. 11:746431.

doi: 10.3389/fonc.2021.746431

\section{Cancer-Related Characteristics Associated With Invasive Mechanical Ventilation or In-Hospital Mortality in Patients With COVID-19 Admitted to ICU: A Cohort Multicenter Study}

Pedro Caruso ${ }^{1,2 *}$, Renato Scarsi Testa ${ }^{1}$, Isabel Cristina Lima Freitas ${ }^{1}$, Ana Paula Agnolon Praça ${ }^{1}$, Valdelis Novis Okamoto $^{1}$, Pauliane Vieira Santana ${ }^{1}$, Ramon Teixeira Costa ${ }^{1}$, Alexandre Melo Kawasaki ${ }^{1,2}$, Renata Rego Lins Fumis ${ }^{3}$, Wilber Antonio Pino Illanes ${ }^{3}$, Eduardo Leite Vieira Costa ${ }^{2,3}$, Thais Dias Midega ${ }^{4}$, Thiago Domingos Correa ${ }^{4}$, Fabrício Rodrigo Torres de Carvalho ${ }^{1,4}$ and Juliana Carvalho Ferreira ${ }^{1,2}$

\footnotetext{
1 Intensive Care Unit, AC Camargo Cancer Center, Sao Paulo, Brazil, 2 Divisao de Pneumologia, Instituto do Coracao (InCor), Hospital das Clinicas HCFMUSP, Faculdade de Medicina, Universidade de Sao Paulo, Sao Paulo, Brazil, ${ }^{3}$ Hospital SírioLibanês, Research and Education Institute, Sao Paulo, Brazil, ${ }^{4}$ Department of Critical Care Medicine, Hospital Israelita Albert Einstein, Sao Paulo, Brazil
}

Background: Coexistence of cancer and COVID-19 is associated with worse outcomes. However, the studies on cancer-related characteristics associated with worse COVID-19 outcomes have shown controversial results. The objective of the study was to evaluate cancer-related characteristics associated with invasive mechanical ventilation use or inhospital mortality in patients with COVID-19 admitted to intensive care unit (ICU).

Methods: We designed a cohort multicenter study including adults with active cancer admitted to ICU due to COVID-19. Seven cancer-related characteristics (cancer status, type of cancer, metastasis occurrence, recent chemotherapy, recent immunotherapy, lung tumor, and performance status) were introduced in a multilevel logistic regression model as first-level variables and hospital was introduced as second-level variable (random effect). Confounders were identified using directed acyclic graphs.

Results: We included 274 patients. Required to undergo invasive mechanical ventilation were 176 patients (64.2\%) and none of the cancer-related characteristics were associated with mechanical ventilation use. Approximately 155 patients died in hospital (56.6\%) and poor performance status, measured with the Eastern Cooperative Oncology Group (ECOG) score was associated with increased in-hospital mortality, with odds ratio $=$ $3.54(1.60-7.88,95 \% \mathrm{Cl})$ for $\mathrm{ECOG}=2$ and odds ratio $=3.40(1.60-7.22,95 \% \mathrm{Cl})$ for $E C O G=3$ to 4 . Cancer status, cancer type, metastatic tumor, lung cancer, and recent chemotherapy or immunotherapy were not associated with in-hospital mortality. 


\begin{abstract}
Conclusions: In patients with active cancer and COVID-19 admitted to ICU, poor performance status was associated with in-hospital mortality but not with mechanical ventilation use. Cancer status, cancer type, metastatic tumor, lung cancer, and recent chemotherapy or immunotherapy were not associated with invasive mechanical ventilation use or in-hospital mortality.
\end{abstract}

Keywords: cancer, COVID-19, intensive care unit, neoplasms, hospital mortality, respiration, artificial

\section{INTRODUCTION}

It has been shown that patients with cancer are more susceptible to the disease caused by the new SARS-CoV-2 virus (COVID-19) $(1,2)$ and that the coexistence of cancer and COVID-19 is associated with worse outcomes, such as hospitalization, invasive mechanical ventilation (MV) use, intensive care unit (ICU) admission, and mortality (1-5). However, the studies that evaluated the cancer-related characteristics associated with worse COVID-19 outcomes have shown controversial results.

In some studies, recent chemotherapy $(6-10)$, recent immunotherapy (11), type of cancer $(3,6,9-12)$, worse performed status $(10,13,14)$, lung as the primary site of the solid tumor $(1,6,12)$, metastatic tumor (3), and the cancer status $(8,10,13,15,16)$ were associated with worse COVID-19 outcomes, while other studies showed that recent chemotherapy $(11,12,14,17-20)$, recent immunotherapy (10, $19)$, type of cancer $(15,21)$, worse performed status $(12,20)$, lung cancer $(3,14,16,17)$, metastatic tumor $(11,12,14,18,19)$, and the cancer status $(8,12,19)$, were not associated with worse outcomes (Supplementary Table $\mathbf{1}$ ).

One plausible explanation for the controversial results is the heterogeneity of the evaluated population. Most studies included outpatients and inpatients in different proportions, including patients with different disease severity, ranging from asymptomatic (20) to hospitalized patients $(3,7,8)$. However, no study evaluated only critically ill patients with active cancer admitted to ICU due to COVID-19, that are the patients with the higher probability of MV use and death.

Better understanding of the cancer-related characteristics associated with worse outcomes can benefit critically ill patients with cancer and COVID-19, providing better ICU triage and, for patients already in the ICU, providing more appropriate therapeutic planning, prognostication and counseling for patients and their relatives.

In critically ill patients with active cancer admitted to ICU due to COVID-19, the objective of the present study was to evaluate the association between cancer-related characteristics and MV use or in-hospital mortality.

\section{METHODS}

Cohort multicenter study included patients from ICUs of four hospitals located in São Paulo, Brazil. The AC Camargo Cancer Center ethics committees approved this study (2521/18L) and waived the need for informed consent.

\section{Patients}

We included consecutive adult patients ( $\geq 18$ years old) with solid tumors or hematologic malignancies and ICU admission due to COVID-19. We excluded patients with cancer remission $\geq 5$ years; decision to forego life-sustaining therapies prior to ICU admission; and admissions for elective postoperative care. If a patient had multiple ICU admissions, only the first was considered. COVID-19 was confirmed by a positive SARSCov-2 real-time reverse transcription polymerase chain reaction in a patient with compatible symptoms or chest computerized tomography findings suggestive of COVID-19.

\section{Data Collection}

Variables were prospectively collected. Each center employed a different data entry form. However, all forms had the same fields and used a standardized definition of the variables.

The following cancer-related characteristics were recorded: 1 . Cancer status related to cancer treatment, categorized as newly diagnosed without treatment, partial or complete response, progressive cancer despite treatment; 2. Type of cancer (solid tumor or hematologic malignancy); 3. Metastatic tumor; 4. Recent chemotherapy or immunotherapy, defined as therapy in the last 30 days; 5 . Lung as the primary site of the solid tumor (pulmonary metastases from other solid tumor sites were not considered as lung cancer); and 6. Performance status, measured with the Eastern Cooperative Oncology Group (ECOG) score.

Upon ICU admission, patient's demographic characteristics, Simplified Acute Physiology Score (SAPS 3) (22), ECOG performance status (23); the Sequential Organ Failure Assessment Score (SOFA) (24); Charlson comorbidity index (25), and specific comorbidities, including arterial hypertension, diabetes, chronic pulmonary disease, heart disease, and overweight or obesity were recorded. We also recorded the following symptoms and exams associated with COVID-19: acute (or acute-on-chronic) cough, fever and myalgia, number of lymphocytes in blood, arterial lactate, and serum creatinine, c-reactive protein and D-dimer.

During ICU stay, the need for oxygen therapy (nasal cannula, oronasal or non-rebreathing mask), MV for more than $>24 \mathrm{~h}$, noninvasive mechanical ventilation (facial mask noninvasive ventilation or high-flow nasal cannula), vasopressors (any dose of noradrenaline, vasopressin, or adrenaline $>1 \mathrm{~h}$ ), and hemodialysis use were recorded. Finally, the in-hospital mortality was recorded.

\section{Statistical Analysis}

Categorical and continuous data were presented as absolute values (percentages) and median (25-75\% interquartile range), respectively. Categorical variables were compared using the 
Chi-square test or Fisher's exact test, as appropriate. Continuous variables were compared using the Mann-Whitney test.

Multilevel logistic regression models were used to determine the cancer-related characteristics associated with MV use or inhospital mortality. The cancer-related characteristics were introduced in the multilevel logistic regression model as firstlevel variables and hospital introduced as second-level variable (random effect) (26). For each cancer-related characteristic, we used a directed acyclic graph to identify confounders (27). Multicollinearity of the confounders was explored using the variance inflation factors and a value $>2.5$ defined collinearity. Odds ratios (OR) and 95\% confidence intervals (CI) were used to measure the association between each variable and MV use or inhospital mortality. If no confounder was identified, the model was adjusted for age and sex.

To estimate the causal inference of the cancer-related characteristics on MV use, the regression models for cancer status, type of cancer, and performance status were adjusted for age, sex, Charlson comorbidity index and lung cancer. The regression models for recent use of chemotherapy or immunotherapy, and lung cancer were adjusted for age, sex, and Charlson comorbidities index. Finally, for metastatic tumor, the directed acyclic graph method did not detect any confounder, therefore the regression model was adjusted for age and sex, as prespecified (Supplementary Figure 1).

To estimate the association between cancer-related characteristics and in-hospital mortality, the regression models of all cancer-related characteristics were adjusted for age, sex, and Charlson comorbidities index (Supplementary Figure 2).

Multicollinearity of the confounders was explored using the variance inflation factors and a value $>2.5$ defined collinearity.

We presented the unadjusted and adjusted odds ratios with 95\% CI for each cancer-related characteristic. As sensitivity analysis, we reproduce the multilevel logistic regression models of the primary analysis, but including SAPS 3 score, as a proxy of the acute illness severity. In an additional sensitivity analysis, we included the number of lymphocytes in blood and c-reactive protein level, as confounders, in the multilevel logistic regression.

There were missing values for arterial lactate $(31.4 \%)$, Ddimer $(36.5 \%)$, and C-reactive protein (5.1\%) upon ICU admission. These missing values were not imputed.

Statistical analyses were performed by SPSS software (Version 23.0. Armonk, NY: IBM Corp). $P$-values $\leq 0.05$ were considered significant. The directed acyclic graphs were created using the browser-based environment DAGitty (28). We followed the recommendations of the STROBE statement that guides the report of observational studies (29) and the guidance for control of confounding and reporting of results in causal inference studies from editors of respiratory, sleep, and critical care journals (27) (See Supplementary Material for further details).

\section{RESULTS}

From February 2020 until November 2020, 274 patients with active cancer and COVID-19 were admitted to the ICU in the participating centers and were included in the study.
The characteristics of the patients upon ICU admission are depicted in Table 1. Upon ICU admission, the hospital nonsurvivors had more organs dysfunction, higher D-dimer and Creactive protein, and lower performance status compared with hospital survivors. Age, sex, type of cancer, site of the primary tumor, and cancer status were similar between survivors and non-survivors. The prevalence of comorbidities was similar between survivors and non-survivors, except for diabetes. No patient received any dose of COVID-19 vaccine.

During ICU stay, hospital non-survivors required more MV, vasopressors, and hemodialysis. The use of oxygen therapy, facial mask noninvasive mechanical ventilation, and high-flow nasal cannula were similar between survivors and non-survivors (Table 2).

\section{Association With Invasive Mechanical Ventilation}

During hospital stay, 176 patients required MV (64.2\%) with a mean duration of 9 days (6-15). Fifty patients (28.4\%) initiated the MV while in ward or emergency room. In-hospital mortality was $27.4 \%(18.6-36.2 \%, 95 \% \mathrm{CI})$ for patients who did not require $\mathrm{MV}$, and $72.7 \%(66.1-79.3 \%, 95 \% \mathrm{CI})$ for patients that required $\mathrm{MV}$.

In a multivariable model adjusting for confounders, none of the cancer-related characteristics were associated with MV use (Figure 1A). The unadjusted models (Supplementary Figure 3) and the sensitivity analyses (Supplementary Figures 4, 5) also showed that cancer-related characteristics were not associated with MV.

\section{Association With In-Hospital Mortality}

Approximately 134 patients died in ICU (48.9\%, 43.0-54.8\%, 95\% CI). Among the 140 patients discharged alive from ICU, 21 died in hospital, resulting an in-hospital mortality of $56.6 \%$ (50.7-62.5\%, 95\% CI).

In the adjusted models, a poor performance status (ECOG $\geq 2$ ) was associated with increased in-hospital mortality (Figure 1B). Metastatic tumor was associated with a twofold increase in the in-hospital mortality, but this estimate was imprecise $[\mathrm{OR}=1.99(0.97-4.07,95 \% \mathrm{CI})]$. Likewise, recent chemotherapy was associated with a 1.7 -fold increase in mortality, but this estimate was imprecise [OR $=1.72(0.94-$ $3.17,95 \% \mathrm{CI})$ ]. Cancer status, cancer type, lung cancer, and recent immunotherapy were not associated with in-hospital mortality. The unadjusted models (Supplementary Figure 6) and the sensitivity analyses (Supplementary Figures 7, 8) showed results similar to the primary analysis.

\section{DISCUSSION}

We showed that cancer-related characteristics were not associated with MV use in critically ill patients with COVID19. We also showed that poor performance status was associated with in-hospital mortality. 
TABLE 1 | Characteristics upon intensive care unit admission of hospital survivors and non-survivors.

\begin{tabular}{|c|c|c|c|}
\hline Variable & Survivors $(n=119)$ & Non-survivors $(n=155)$ & $\boldsymbol{P}$ \\
\hline Male & $74(62.2)$ & $85(54.8)$ & 0.27 \\
\hline Age (years) & $66(58-75)$ & $64(52-74)$ & 0.18 \\
\hline \multicolumn{4}{|l|}{ Comorbidities } \\
\hline Arterial hypertension & $62(52.1)$ & $70(45.2)$ & 0.27 \\
\hline Diabetes & $42(35.3)$ & $27(17.4)$ & $<0.01$ \\
\hline COPD & $15(12.6)$ & $16(10.3)$ & 0.57 \\
\hline Heart disease & $18(15.1)$ & $16(10.3)$ & 0.27 \\
\hline $\mathrm{BMl}>25 \mathrm{~kg} / \mathrm{m}^{2}$ & $60(50.4)$ & $66(42.6)$ & 0.22 \\
\hline Chronic kidney failure & $12(10.1)$ & $12(7.7)$ & 0.52 \\
\hline Charlson comorbidities index & $6(3-7)$ & $6(4-8)$ & 0.15 \\
\hline SAPS 3 & $58(49-64)$ & $71(59-82)$ & $<0.01$ \\
\hline \multirow[t]{2}{*}{ SOFA } & $1(0-3)$ & $4(2-7)$ & $<0.01$ \\
\hline & \multicolumn{3}{|c|}{ COVID-19 characteristics } \\
\hline \multicolumn{4}{|l|}{ COVID-19 symptoms } \\
\hline Fever & $68(57.1)$ & $68(43.9)$ & 0.03 \\
\hline Acute cough & $71(59.7)$ & $79(51.0)$ & 0.14 \\
\hline Breathless & $76(63.9)$ & $118(76.1)$ & 0.03 \\
\hline Myalgia & $20(16.8)$ & $17(11.0)$ & 0.21 \\
\hline \multicolumn{4}{|l|}{ Laboratory results } \\
\hline Arterial lactate $(\mathrm{mg} / \mathrm{dl})(\mathrm{n}=188)$ & $14(10-19)$ & $16(12-23)$ & 0.02 \\
\hline D-dimer (ng/ml) (n = 174) & $1,158(690-1,949)$ & 2,355 (1,093-6,337) & $<0.01$ \\
\hline Lymphocyte (cells per mm³) & $720(450-1,180)$ & $585(302-1,032)$ & 0.05 \\
\hline C-reactive protein $(\mathrm{mg} / \mathrm{l})(\mathrm{n}=260)$ & $10(5-26)$ & $18(12-27)$ & $<0.01$ \\
\hline \multirow[t]{2}{*}{ Creatinine (mg/dl) } & $0.96(0.70-1.36)$ & $1.14(0.71-1.78)$ & 0.04 \\
\hline & \multicolumn{3}{|c|}{ Cancer-related characteristics } \\
\hline Type of cancer & & & 0.88 \\
\hline Hematologic malignancy & $27(22.7)$ & $33(20.6)$ & \\
\hline Solid tumor & $92(77.3)$ & $122(78.7)$ & \\
\hline Metastatic tumor & $36(30.3)$ & $69(44.5)$ & 0.01 \\
\hline Type of hematologic malignancy & & & 0.99 \\
\hline Low-grade hematologic malignancy & $18(15.1)$ & $22(14.2)$ & \\
\hline High-grade hematologic malignancy & $9(7.6)$ & $11(7.1)$ & \\
\hline Site of solid tumor & & & 0.26 \\
\hline Prostate & $21(17.6)$ & $14(9.0)$ & \\
\hline Breast & $16(13.4)$ & $25(16.1)$ & \\
\hline Lung & $6(5.0)$ & $14(9.0)$ & \\
\hline Head and neck & $6(5,0)$ & $6(3.9)$ & \\
\hline Colon & $7(5.9)$ & $12(7.7)$ & \\
\hline Bladder & $3(2.5)$ & $7(4.5)$ & \\
\hline Melanoma & $6(5.0)$ & $3(2.0)$ & \\
\hline Others & $27(22.7)$ & $42(27.1)$ & \\
\hline Lung cancer & $6(5.0)$ & $14(9.0)$ & 0.25 \\
\hline Cancer status & & & 0.09 \\
\hline Newly diagnosed without treatment & $8(6.7)$ & $18(11.6)$ & \\
\hline Partial or complete response & $78(65.5)$ & $82(52.9)$ & \\
\hline Progressive & $33(27.7)$ & $55(35.5)$ & \\
\hline \multicolumn{4}{|l|}{ Previous cancer treatment } \\
\hline Surgery & $67(56.3)$ & $74(47.7)$ & 0.14 \\
\hline Chemotherapy & $71(59.7)$ & $107(69.0)$ & 0.08 \\
\hline Recent chemotherapy (<30 days) & $23(19.3)$ & $44(28.4)$ & 0.12 \\
\hline Immunotherapy & $12(10.1)$ & $15(9.7)$ & 0.99 \\
\hline Recent Immunotherapy (<30 days) & $7(5.9)$ & $8(5.2)$ & 0.79 \\
\hline Radiotherapy & $36(30.3)$ & $49(31.6)$ & 0.99 \\
\hline Performance status (ECOG) & & & $<0.01$ \\
\hline $0-1$ & $95(79.8)$ & $86(55.5)$ & \\
\hline 2 & $10(8.4)$ & $35(22.6)$ & \\
\hline $3-4$ & $13(10.8)$ & $34(21.9)$ & \\
\hline
\end{tabular}

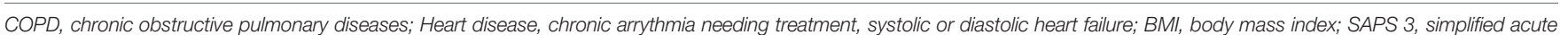

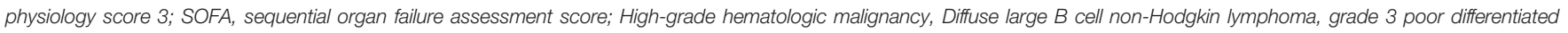

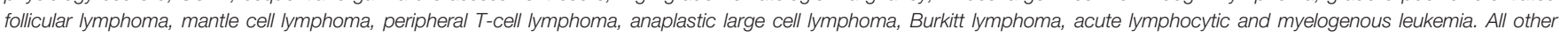
hematologic malignancies were considered as low-grade; ECOG, Eastern Cooperative Oncology Group.

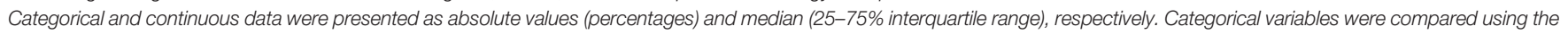
Chi-square test or Fisher's exact test, as appropriate. Continuous variables were compared using the Mann-Whitney test. 
TABLE 2 | Characteristics during ICU stay of hospital survivors and non-survivors.

\begin{tabular}{|c|c|c|c|}
\hline Variable & Survivors $(n=119)$ & Non-survivors $(n=155)$ & $P$ \\
\hline Oxygen therapy & $112(94.1)$ & $149(96.1)$ & 0.77 \\
\hline High-flow nasal cannula & $15(12.6)$ & $18(11.6)$ & 0.85 \\
\hline Facial mask noninvasive MV & $35(29.4)$ & $43(27.7)$ & 0.99 \\
\hline Invasive MV & $48(40.3)$ & $128(82.6)$ & $<0.01$ \\
\hline Vasopressors & $50(42.0)$ & $130(83.9)$ & $<0.01$ \\
\hline Hemodialysis & $14(11.8)$ & $56(36.1)$ & $<0.01$ \\
\hline Invasive MV duration (days) & $8(5-12)$ & $10(6-16)$ & 0.11 \\
\hline Ventilator-free (days) & $6.0(3.0-9.3)$ & $1.0(0.0-3.0)$ & $<0.01$ \\
\hline ICU length of stay (days) & $5(3-14)$ & $9(4-17)$ & 0.02 \\
\hline Hospital length of stay (days) & $22(13-33)$ & $16(9-24)$ & $<0.01$ \\
\hline
\end{tabular}

MV, mechanical ventilation; Vasopressors, noradrenaline, vasopressin, or adrenaline $>1 \mathrm{~h}$.

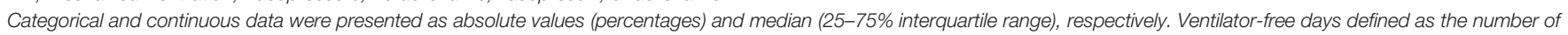
days alive and free from mechanical ventilation for at least 48 consecutive hours.

Categorical variables were compared using the Chi-square test or Fisher's exact test, as appropriate. Continuous variables were compared using the Mann-Whitney t-test.

The association of cancer-related characteristics with worse outcomes in patients with COVID-19 is controversial, probably because cancer and COVID-19 are diseases with large heterogeneity, that combined with methodological differences turned the studies barely comparable. Moreover, the studies evaluated different populations. Considering the age, the studies included adults, children or a mixed sample of adult and pediatric patients $(18,30)$. Considering the type of tumor, studies included patients with solid tumors (7), specific solid tumors (lung or thoracic) $(19,20)$, hematologic malignancies (8), or patients with solid tumors and hematological malignancies with different proportions $(12,18)$. Considering the COVID-19 aspects, studies included outpatients and inpatients with different levels of COVID-19 severity, including asymptomatic patients (20), patients that went to hospital for scheduled cancer treatment (15), and hospitalized patients (8). The primary outcomes also varied among the studies $(8,15,17,20)$ and the same outcome, such as mortality, had different definitions $(8,9$, $13,18,30)$. Regional care differences and SARS-CoV-2 variants may also have contributed to the differences. Nationwide studies showed in-hospital mortality varying from $17 \%$ in Germany (31) to $38 \%$ in Brazil (32), probably reflecting differences in healthcare system, adherence to best practices, and temporal spread of the pandemic. Some studies were performed in United Kingdom (9) or Brazil (16), where the British Alpha (former B.1.1.7) $(33,34)$ and the Brazilian Gamma (former P.1) (35) variants are predominant, more transmissible and have an unknown impact on outcomes $(33,34)$. Finally, the studies employed different methodologies and most included preestablished or $P$-based select confounders, what is not in accordance with the best practices to control of confounding and reporting of results in causal inference studies (27).

It is known that patients with cancer are more susceptible to COVID-19 (1, 2) and have worse outcomes, such as hospitalization and ICU admission (1-5). However, for patients admitted with severe COVID-19, cancer-related characteristics may lose or decrease their impact on need for MV and mortality, as we showed in the present study. A similar finding was reported by Brar et al. in a study that compared hospitalized COVID-19infected patients without cancer with a matched for cohort of patients with COVID-19 and cancer, showing that cancer was not associated with need for MV or mortality (36).

In the present study, cancer-related characteristics were not associate with MV use. Limited mechanistic data are available on
A

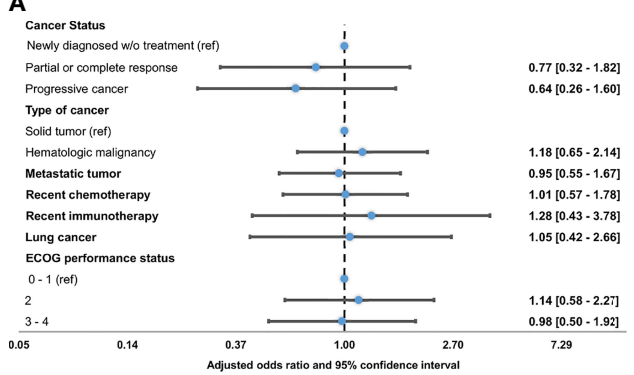

B

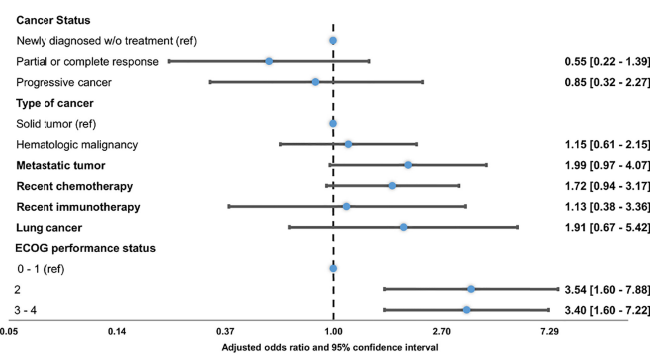

FIGURE 1 | Forest plot of cancer-related characteristics associated with invasive mechanical ventilation use (A) or in-hospital mortality (B). Data are adjusted odds ratios with 95\% confidence intervals. ECOG, Eastern Cooperative Oncology Group. Panel (A) (invasive mechanical ventilation use): Cancer status, type of cancer, and performance status were adjusted for age, sex, Charlson comorbidity index and lung cancer. Recent use of chemotherapy or immunotherapy, and lung cancer were adjusted for age, sex and Charlson comorbidities index. Metastatic tumor was adjusted for age and sex. Panel (B) (in-hospital mortality): All models adjusted for age, sex, and Charlson comorbidities index. 
the causes of COVID-19 progression to acute respiratory distress syndrome (ARDS). We know that SARS-CoV-2 has specific virulence mechanisms that, combined with personal innate antiviral response, account for the heterogeneous clinical evolution of patients with COVID-19 (37, 38). This combination, and not the presence of cancer, may be the determinants of progression to moderate or severe ARDS. Corroborating this hypothesis, $\mathrm{Xu}$ et al., using a machine learning model, showed that malignancy was not associated with progression to ARDS in patients with COVID-19 (39).

In the present study, no cancer-related characteristic, except performance status, was associated with in-hospital mortality. One could expect that hematologic malignancies (type of cancer) would be associated with higher in-hospital mortality because critically ill patients with hematologic malignancies have presented higher short-term mortality compared with patients with solid tumors (40). However, our results did not show an association of hematologic malignancies with in-hospital mortality. The first hypothesis for this discrepancy is that, in previous studies (40), patients with hematologic malignancies were admitted to ICU for cancer or treatment-related complications, while during the pandemic these patients were admitted for an acute respiratory viral disease. The second hypothesis is that, in the last years, patients with hematologic malignancies had higher reductions in short-term mortality than patients with solid tumors (41). Finally, a study that evaluated inhospital mortality of patients with cancer admitted to ICU requiring ventilatory support showed that hematologic malignancies were not associated with mortality (42). We also could expect that recent chemotherapy and immunotherapy could impact on in-hospital mortality. However, our results did not show an association of recent cancer treatment with in-hospital mortality. Recent chemotherapy weakens the immune system, while recent immunotherapy enhances it. A weakened immune system could negatively impact on outcomes increasing viral replication but could positively impact avoiding the cytokine storm. On the other hand, an enhanced immune system could positively impact on outcomes decreasing viral replication, but negatively promoting the cytokine storm. Therefore, the impact of recent chemotherapy and immunotherapy can vary unexpected and in opposite direction.

We showed that poor performance status was associated with in-hospital mortality. Poor performance status has been associated with in-hospital mortality in patients with cancer and acute respiratory failure due to COVID-19 (14) or other causes (42). Zampieri et al. showed that poor performance status was associated with increased in-hospital mortality of critically ill patients, regardless of the cancer-related characteristics (43). Two studies did not show the association of poor performance status with worse outcomes in patients with cancer and COVID19 (12, 20). However, both studies mixed outpatients with inpatients, with COVID-19 severity ranging from asymptomatic to critically-ill patients, and one study evaluated only patients with thoracic tumors (20).

In critically ill patients with COVID-19, the knowledge of cancer-related characteristics associated with worse outcomes can be used to prompt a closer monitoring, avoiding ICU admission delay, which has been associated with higher mortality in patients with cancer and acute respiratory failure (44). Additionally, in patients with progressive cancer despite treatment, the knowledge can allow better ICU triage, following the principles of beneficence, non-maleficence, and autonomy (45). Information about the cancer-related characteristics associated with worse outcomes are important for providing proper prognostication, therapeutic planning and counseling for all patients and their relatives. Finally, our findings suggest that cancer-related characteristics should not motivate intensive care unit triage and treatment decisions, except when performance status is poor.

Our study has limitations inherent to observational studies. The care of patients across different centers might not be comparable. However, we employed a statistical analysis designed to deal with heterogeneity among centers (26), and the heterogeneous care reflects real-world conditions. Although consecutive inclusion mitigates the risk of selection bias, we excluded critically ill patients with cancer and COVID-19 that were not admitted to the ICU due to perceived futility of ICU admission and it is possible that those patients had more metastatic disease, progressive cancer and poor performance status. In addition, our sample size may have been insufficient to detect the association between cancer-related characteristics, such as metastatic cancer and recent chemotherapy with mortality.

\section{CONCLUSIONS}

In patients with active cancer admitted to ICU due to COVID19 , a poor performance status is associated with increased inhospital mortality. Metastatic tumor and recent chemotherapy are associated with approximately twofold increase in hospital mortality, but the estimates are imprecise. The cancer status, type of cancer, lung cancer, and recent immunotherapy are not associated with increased in-hospital mortality. Cancer-related characteristics are not associated with increased invasive mechanical ventilation use. The results, however, should be cautiously interpreted due to the observational design of the study.

\section{DATA AVAILABILITY STATEMENT}

The raw data supporting the conclusions of this article will be made available by the authors, without undue reservation.

\section{ETHICS STATEMENT}

The AC Camargo Cancer Center ethics committees approved this study (2521/18L) and waived the need for informed consent. Written informed consent for participation was not required for 
this study in accordance with the national legislation and the institutional requirements.

\section{AUTHOR CONTRIBUTIONS}

PC, RT, and AP: Substantial contributions to the conception of the work, acquisition, and interpretation of data. Revised the manuscript critically for important intellectual content. IF, VO, PS, RC, AK, RF, WP, EC, TM, FC, and JF: Acquisition and interpretation of data and revised the manuscript critically for important intellectual content. All authors contributed to the article and approved the submitted version.

\section{REFERENCES}

1. Yang L, Chai P, Yu J, Fan X. Effects of Cancer on Patients With COVID-19: A Systematic Review and Meta-Analysis of 63,019 Participants. Cancer Biol Med (2021) 18(1):298-307. doi: 10.20892/j.issn.2095-3941.2020.0559

2. Liang W, Guan W, Chen R, Wang W, Li J, Xu K, et al. Cancer Patients in SARS-CoV-2 Infection: A Nationwide Analysis in China. Lancet Oncol (2020) 21(3):335-7. doi: 10.1016/S1470-2045(20)30096-6

3. Dai M, Liu D, Liu M, Zhou F, Li G, Chen Z, et al. Patients With Cancer Appear More Vulnerable to SARS-CoV-2: A Multicenter Study During the COVID-19 Outbreak. Cancer Discovery (2020) 10(6):783-91. doi: 10.1158/ 2159-8290.CD-20-0422

4. Elkrief A, Desilets A, Papneja N, Cvetkovic L, Groleau C, Lakehal YA, et al. High Mortality Among Hospital-Acquired COVID-19 Infection in Patients With Cancer: A Multicentre Observational Cohort Study. Eur J Cancer (2020) 139:181-7. doi: 10.1016/j.ejca.2020.08.017

5. Giannakoulis VG, Papoutsi E, Siempos II. Effect of Cancer on Clinical Outcomes of Patients With COVID-19: A Meta-Analysis of Patient Data. JCO Glob Oncol (2020) 6:799-808. doi: 10.1200/GO.20.00225

6. Larfors G, Pahnke S, State M, Fredriksson K, Pettersson D. Covid-19 Intensive Care Admissions and Mortality Among Swedish Patients With Cancer. Acta Oncol (2021) 60(1):32-4. doi: 10.1080/0284186X.2020.1854481

7. Zhang L, Zhu F, Xie L, Wang C, Wang J, Chen R, et al. Clinical Characteristics of COVID-19-Infected Cancer Patients: A Retrospective Case Study in Three Hospitals Within Wuhan, China. Ann Oncol (2020) 31(7):894-901. doi: 10.1016/j.annonc.2020.03.296

8. Cattaneo C, Daffini R, Pagani C, Salvetti M, Mancini V, Borlenghi E, et al. Clinical Characteristics and Risk Factors for Mortality in Hematologic Patients Affected by COVID-19. Cancer (2020) 126(23):5069-76. doi: $10.1002 / \mathrm{cncr} .33160$

9. Lee LYW, Cazier JB, Starkey T, Briggs SEW, Arnold R, Bisht V, et al. COVID19 Prevalence and Mortality in Patients With Cancer and the Effect of Primary Tumour Subtype and Patient Demographics: A Prospective Cohort Study [Published Correction Appears in Lancet Oncol. 2020 Sep 3];. Lancet Oncol (2020) 21(10):1309-16. doi: 10.1016/S1470-2045(20)30442-3

10. Grivas P, Khaki AR, Wise-Draper TM, French B, Hennessy C, Hsu CY, et al. Association of Clinical Factors and Recent Anticancer Therapy With COVID19 Severity Among Patients With Cancer: A Report From the COVID-19 and Cancer Consortium. Ann Oncol (2021) 32(6):787-800. doi: 10.1016/ j.annonc.2021.02.024

11. Robilotti EV, Babady NE, Mead PA, Rolling T, Perez-Johnston R, Bernardes $\mathrm{M}$, et al. Determinants of COVID-19 Disease Severity in Patients With Cancer. Nat Med (2020) 26(8):1218-23. doi: 10.1038/s41591-020-0979-0

12. Jee J, Foote MB, Lumish M, Stonestrom AJ, Wills B, Narendra V, et al. Chemotherapy and COVID-19 Outcomes in Patients With Cancer. J Clin Oncol (2020) 38(30):3538-46. doi: 10.1200/JCO.20.01307

13. Kuderer NM, Choueiri TK, Shah DP, Shyr Y, Rubinstein SM, ShRiveraah DR, et al. Clinical Impact of COVID-19 on Patients With Cancer (CCC19): A Cohort Study [Published Correction Appears in Lancet. 2020 Sep 12;396 (10253):758]. Lancet (2020) 395(10241):1907-18. doi: 10.1016/S0140-6736 (20)31187-9

\section{FUNDING}

This study was funded by the intensive care unit of the AC Camargo Cancer Center.

\section{SUPPLEMENTARY MATERIAL}

The Supplementary Material for this article can be found online at: https://www.frontiersin.org/articles/10.3389/fonc.2021. 746431/full\#supplementary-material

14. Lièvre A, Turpin A, Ray-Coquard I, Le Malicot K, Thariat J, Ahle G, et al. Risk Factors for Coronavirus Disease 2019 (COVID-19) Severity and Mortality Among Solid Cancer Patients and Impact of the Disease on Anticancer Treatment: A French Nationwide Cohort Study (GCO-002 CACOVID-19). Eur J Cancer (2020) 141:62-81. doi: 10.1016/j.ejca.2020.09.035

15. Russell B, Moss C, Papa S, Irshad S, Ross P, Spicer J, et al. Factors Affecting COVID-19 Outcomes in Cancer Patients: A First Report From Guy's Cancer Center in London. Front Oncol (2020) 10:1279. doi: 10.3389/fonc.2020.01279

16. Ferrari BL, Ferreira CG, Menezes M, De Marchi P, Canedo J, Melo AC, et al. Determinants of COVID-19 Mortality in Patients With Cancer From a Community Oncology Practice in Brazil. JCO Glob Oncol (2021) 7:46-55. doi: $10.1200 / G O .20 .00444$

17. Yarza R, Bover M, Paredes D, López-López F, Jara-Casas D, Castelo-Loureiro A, et al. SARS-CoV-2 Infection in Cancer Patients Undergoing Active Treatment: Analysis of Clinical Features and Predictive Factors for Severe Respiratory Failure and Death. Eur J Cancer (2020) 135:242-50. doi: 10.1016/ j.ejca.2020.06.001

18. Mehta V, Goel S, Kabarriti R, Cole D, Goldfinger M, Acuna-Villaorduna A, et al. Case Fatality Rate of Cancer Patients With COVID-19 in a New York Hospital System. Cancer Discovery (2020) 10(7):935-41. doi: 10.1158/21598290.CD-20-0516

19. Luo J, Rizvi H, Preeshagul IR, Egger JV, Hoyos D, Bandlamudi C, et al. COVID-19 in Patients With Lung Cancer. Ann Oncol (2020) 31(10):1386-96. doi: 10.1016/j.annonc.2020.06.007

20. Garassino MC, Whisenant JG, Huang LC, Trama A, Torri V, Agustoni F, et al. COVID-19 in Patients With Thoracic Malignancies (TERAVOLT): First Results of an International, Registry-Based, Cohort Study. Lancet Oncol (2020) 21(7):914-22. doi: 10.1016/S1470-2045(20)30314-4

21. Shoumariyeh K, Biavasco F, Ihorst G, Rieg S, Nieters A, Kern WV, et al. Covid-19 in Patients With Hematological and Solid Cancers at a Comprehensive Cancer Center in Germany. Cancer Med (2020) 9 (22):8412-22. doi: 10.1002/cam4.3460

22. Moreno RP, Metnitz PG, Almeida E, Jordan B, Bauer P, Campos RA, et al. SAPS 3-From Evaluation of the Patient to Evaluation of the Intensive Care Unit. Part 2: Development of a Prognostic Model for Hospital Mortality at ICU Admission [Published Correction Appears in Intensive Care Med. 2006 May;32(5):796]. Intensive Care Med (2005) 31(10):1345-55. doi: 10.1007/ s00134-005-2763-5

23. Oken MM, Creech RH, Tormey DC, Horton J, Davis TE, McFadden ET, et al. Toxicity and Response Criteria of the Eastern Cooperative Oncology Group. Am J Clin Oncol (1982) 5(6):649-55. doi: 10.1097/00000421-19821200000014

24. Vincent JL, Moreno R, Takala J, Willatts S, De Mendonça A, Bruining H, et al. The SOFA (Sepsis-Related Organ Failure Assessment) Score to Describe Organ Dysfunction/Failure. On Behalf of the Working Group on SepsisRelated Problems of the European Society of Intensive Care Medicine. Intensive Care Med (1996) 22(7):707-10. doi: 10.1007/BF01709751

25. Charlson ME, Pompei P, Ales KL, MacKenzie CR. A New Method of Classifying Prognostic Comorbidity in Longitudinal Studies: Development and Validation. J Chronic Dis (1987) 40(5):373-83. doi: 10.1016/0021-9681 (87)90171-8 
26. Diez-Roux AV. Multilevel Analysis in Public Health Research. Annu Rev Public Health (2000) 21:171-92. doi: 10.1146/annurev.publhealth.21.1.171

27. Lederer DJ, Bell SC, Branson RD, Chalmers JD, Marshall R, Maslove DM, et al. Control of Confounding and Reporting of Results in Causal Inference Studies. Guidance for Authors From Editors of Respiratory, Sleep, and Critical Care Journals [Published Correction Appears in Ann Am Thorac Soc. 2019 Feb;16(2):283]. Ann Am Thorac Soc (2019) 16(1):22-8. doi: 10.1513/AnnalsATS.201808-564PS

28. Textor J, van der Zander B, Gilthorpe MS, Liskiewicz M, Ellison GT. Robust Causal Inference Using Directed Acyclic Graphs: The R Package 'Dagitty'. Int J Epidemiol (2016) 45(6):1887-94. doi: 10.1093/ije/dyw341

29. von Elm E, Altman DG, Egger M, Pocock SJ, Gøtzsche PC, Vandenbroucke JP, et al. The Strengthening the Reporting of Observational Studies in Epidemiology (STROBE) Statement: Guidelines for Reporting Observational Studies [Published Correction Appears in Ann Intern Med. 2008 Jan 15;148 (2):168]. Ann Intern Med (2007) 147(8):573-7. doi: 10.7326/0003-4819-1478-200710160-00010

30. Piñana JL, Martino R, García-García I, Parody R, Morales MD, Benzo G, et al. Risk Factors and Outcome of COVID-19 in Patients With Hematological Malignancies. Exp Hematol Oncol (2020) 9:21. doi: 10.1186/s40164-020-00177-z

31. Karagiannidis C, Mostert C, Hentschker C, Voshaar T, Malzahn J, Schillinger G, et al. Case Characteristics, Resource Use, and Outcomes of 10021 Patients With COVID-19 Admitted to 920 German Hospitals: An Observational Study. Lancet Respir Med (2020) 8(9):853-62. doi: 10.1016/ S2213-2600(20)30316-7

32. Ranzani OT, Bastos LSL, Gelli JGM, Marchesi JF, Baião F, Hamacher S, et al. Characterisation of the First 250,000 Hospital Admissions for COVID-19 in Brazil: A Retrospective Analysis of Nationwide Data. Lancet Respir Med (2021) 9(4):407-18. doi: 10.1016/S2213-2600(20)30560-9

33. Davies NG, Abbott S, Barnard RC, Jarvis CI, Kucharski AJ, Munday JD, et al. Estimated Transmissibility and Impact of SARS-CoV-2 Lineage B.1.1.7 in England. Science (2021) 372(6538):eabg3055. doi: 10.1126/science.abg3055

34. Frampton D, Rampling T, Cross A, Bailey H, Heaney J, Byott M, et al. Genomic Characteristics and Clinical Effect of the Emergent SARS-CoV-2 B.1.1.7 Lineage in London, UK: A Whole-Genome Sequencing and Hospital-Based Cohort Study. Lancet Infect Dis (2021) 21(9):1246-56. doi: 10.1016/S1473-3099(21)00170-5

35. Faria NR, Mellan TA, Whittaker C, Claro IM, Candido DDS, Mishra S, et al. Genomics and Epidemiology of the P.1 SARS-CoV-2 Lineage in Manaus, Brazil. Science (2021) 372(6544):815-21. doi: 10.1126/science.abh2644

36. Brar G, Pinheiro LC, Shusterman M, Swed B, Reshetnyak E, Soroka O, et al. COVID-19 Severity and Outcomes in Patients With Cancer: A Matched Cohort Study. J Clin Oncol (2020) 38(33):3914-24. doi: 10.1200/JCO.20.01580

37. Torres Acosta MA, Singer BD. Pathogenesis of COVID-19-Induced ARDS: Implications for an Ageing Population. Eur Respir J (2020) 56(3):2002049. doi: 10.1183/13993003.02049-2020

38. Attaway AH, Scheraga RG, Bhimraj A, Biehl M, Hatipoğlu U. Severe Covid-19 Pneumonia: Pathogenesis and Clinical Management. BMJ (2021) 372:n436. doi: 10.1136/bmj.n436
39. Xu W, Sun NN, Gao HN, Chen ZY, Yang Y, Ju B, et al. Risk Factors Analysis of COVID-19 Patients With ARDS and Prediction Based on Machine Learning. Sci Rep (2021) 11(1):2933. doi: 10.1038/s41598-021-82492-x

40. Taccone FS, Artigas AA, Sprung CL, Moreno R, Sakr Y, Vincent JL. Characteristics and Outcomes of Cancer Patients in European ICUs. Crit Care (2009) 13(1):R15. doi: 10.1186/cc7713

41. Zampieri FG, Romano TG, Salluh JIF, Taniguchi LU, Mendes PV, Nassar AP Jr, et al. Trends in Clinical Profiles, Organ Support Use and Outcomes of Patients With Cancer Requiring Unplanned ICU Admission: A Multicenter Cohort Study [Published Correction Appears in Intensive Care Med. 2020 Oct 2];. Intensive Care Med (2021) 47(2):170-9. doi: 10.1007/s00134-020-06184-2

42. Azevedo LCP, Caruso P, Silva UVA, Torelly AP, Silva E, Rezende E, et al. Outcomes for Patients With Cancer Admitted to the ICU Requiring Ventilatory Support: Results From a Prospective Multicenter Study. Chest (2014) 146(2):257-66. doi: 10.1378/chest.13-1870

43. Zampieri FG, Bozza FA, Moralez GM, Mazza DD, Scotti AV, Santino MS, et al. The Effects of Performance Status One Week Before Hospital Admission on the Outcomes of Critically Ill Patients. Intensive Care Med (2017) 43 (1):39-47. doi: 10.1007/s00134-016-4563-5

44. Mokart D, Lambert J, Schnell D, Fouché L, Rabbat A, Kouatchet A, et al. Delayed Intensive Care Unit Admission Is Associated With Increased Mortality in Patients With Cancer With Acute Respiratory Failure. Leuk Lymphoma (2013) 54(8):1724-9. doi: 10.3109/10428194.2012.753446

45. Blot F, Dumont SN, Vigouret-Viant L, Verotte N, Rossignol J, Rieutord A, et al. Ethical Issues Related to the COVID-19 Pandemic in Patients With Cancer: Experience and Organisations in a French Comprehensive Cancer Centre [Published Online Ahead of Print, 2020 Aug 27]. BMJ Support Palliat Care (2020) 27:bmjspcare-2020-002504. doi: 10.1136/bmjspcare-2020-002504

Conflict of Interest: The authors declare that the research was conducted in the absence of any commercial or financial relationships that could be construed as a potential conflict of interest.

Publisher's Note: All claims expressed in this article are solely those of the authors and do not necessarily represent those of their affiliated organizations, or those of the publisher, the editors and the reviewers. Any product that may be evaluated in this article, or claim that may be made by its manufacturer, is not guaranteed or endorsed by the publisher.

Copyright $\odot 2021$ Caruso, Testa, Freitas, Praça, Okamoto, Santana, Costa, Kawasaki, Fumis, Pino Illanes, Costa, Midega, Correa, de Carvalho and Ferreira. This is an open-access article distributed under the terms of the Creative Commons Attribution License (CC BY). The use, distribution or reproduction in other forums is permitted, provided the original author(s) and the copyright owner(s) are credited and that the original publication in this journal is cited, in accordance with accepted academic practice. No use, distribution or reproduction is permitted which does not comply with these terms. 